\title{
Advances in coherent detection algorithms
}

Jens Rasmussen, Takeshi Hoshida, Takahito Tanimura, Hisao Nakashima, Shoichiro Oda, et al.

Jens C. Rasmussen, Takeshi Hoshida, Takahito Tanimura, Hisao Nakashima, Shoichiro Oda, Zhenning Tao, Lei Li, "Advances in coherent detection algorithms," Proc. SPIE 7960, Coherent Optical Communication:

Components, Subsystems, and Systems, 796002 (25 January 2011); doi: 10.1117/12.881627

SPIE. Event: SPIE OPTO, 2011, San Francisco, California, United States 


\title{
Advances in coherent detection algorithms
}

\author{
Jens C. Rasmussen*a, Takeshi Hoshida ${ }^{\mathrm{a}}$, Takahito Tanimura ${ }^{\mathrm{a}}$, Hisao Nakashima ${ }^{\mathrm{a}}$, Shoichiro Oda ${ }^{\mathrm{a}}$, \\ Zhenning $\mathrm{Tao}^{\mathrm{b}}$, Lei $\mathrm{Li}^{\mathrm{b}}$ \\ ${ }^{a}$ Fujitsu Laboratories Ltd., 1-1 Kamikodanaka 4-chome, Nakahara-ku, Kawasaki 211-8588, Japan \\ ${ }^{b}$ Fujitsu R\&D Center, 13/F Tower A, No. 56 Dong Si Huan Zhong Rd, Beijing, 100025, China
}

\begin{abstract}
Digital coherent receivers with data-rates of $100 \mathrm{Gbit} / \mathrm{s}$ based on dual-polarization quaternary phase shift keying (DPQPSK) have become a reality. One research trend is now directing towards even higher bit-rates of $400 \mathrm{Gbit} / \mathrm{s}$ and 1 Tbit/s. However, it is also very desirable to improve the performance of the current basic $100 \mathrm{Gbit} / \mathrm{s}$ DP-QPSK. Algorithms have a huge improvement potential and exemplary recent advances will be introduced in this paper.
\end{abstract}

Keywords: digital coherent receiver, algorithm, dual-polarization, QPSK

\section{INTRODUCTION}

The principal advantages of coherent receivers are well known. However and although initial studies that form the basis of today's renewed interest date back as early as 1990's [1]-[3], it wasn't until recently, i.e. about two decades later that real-time operating receivers were reported initially FPGA based with bit-rates up to $10 \mathrm{Gbit} / \mathrm{s}$ and followed by ASIC implementations with bit-rates of $40 \mathrm{Gbit} / \mathrm{s}$ and above [4]-[7], [15]. Up to data-rates of $10 \mathrm{Gbit} / \mathrm{s}$, which were introduced more than about a decade ago and nowadays are mainstream, direct detection receivers provided a sufficient performance in terms of achievable reach and dispersion tolerance while allowing for economic realizations based on mainly on-off keying (OOK) modulation format. Data-rates above $10 \mathrm{Gbit} / \mathrm{s}$ rely on advanced modulation formats to achieve a reasonable reach, dispersion tolerance, and spectral efficiency necessary to support transport within the limited optical bandwidth of today's dense-wavelength division multiplexed (DWDM) systems with $50 \mathrm{GHz}$ channel spacing. For $100 \mathrm{Gbit} / \mathrm{s}$, the industry settled on dual-polarization quaternary phase shift keying (DP-QPSK) as the modulation format of choice supported by OIF implementation agreements. A DP-QPSK digital coherent receiver splits the optical data carrying signal into two orthogonal polarizations. Optical hybrids are used to mix these signals with light of a local oscillator laser with a frequency close but not necessarily equal to the carrier frequency (intradyne detection) of the optical signal [3]. The resultant 4 optical signals, inphase and quadrature components of the two polarizations, are digitized by analog-to-digital converters and processed in the digital domain. Digital coherent receiver algorithms can be divided into those that are fundamentally necessary like carrier phase recovery, and those that provide enhancing or additional functionality like digital skew compensation [8] or non-linearities mitigation [9]-[11]. The basic digital coherent receiver and its basic algorithms are briefly introduced in section 2. Exemplary advanced algorithms are discussed in section 3 and section 4 provides a summary.

\section{DIGITAL COHERENT RECEIVER}

A digital coherent receiver typically consists of a polarization diversity front-end, analog-to-digital (A/D) converters, and a digital signal processing part. The received optical signal is split into two orthogonal polarization components, each of which is mixed with the corresponding polarization of a local oscillator laser by means of optical hybrids. Inphase and quadrature signals of each polarization are then digitized by A/D converters. Digital signal processing then follows to perform the following generic operations (Fig. 1).

*jens.rasmussen@jp.fujitsu.com; phone +81 44 754-2641; fax +81 44 754-2640; jp.fujitsu.com/labs 
- Quasi-static equalization

Chromatic dispersion is compensated either in the time domain by means of a finite-impulse-response filter (FIR) or in the frequency domain by means of Fourier- and inverse Fourier transformations (FFT/iFFT). With increasing chromatic dispersion, a frequency domain realization becomes more and more advantageous in terms of implementation complexity [12]. Chromatic dispersion of an optical fiber changes slightly with temperature [13] but there are no rapid changes and fast adaption is not required. The small chromatic dispersion variations can usually be compensated for by the following adaptive equalizer.

- Adaptive equalization and polarization recovery

During transmission through optical fibers and components, the signal polarization changes and the state of polarization at the receiver is not known. Polarization de-multiplexing/recovery can be performed using a $2 \times 2$ multiple-input-multiple-output (MIMO) equalizer. Decision-directed [14] and constant-modulus algorithm (CMA) based [15] methods have been proposed. If the equalizer is realized based on finite-impulse response filters (FIR), it can compensate for also polarization mode dispersion (PMD) and the residual chromatic dispersion.

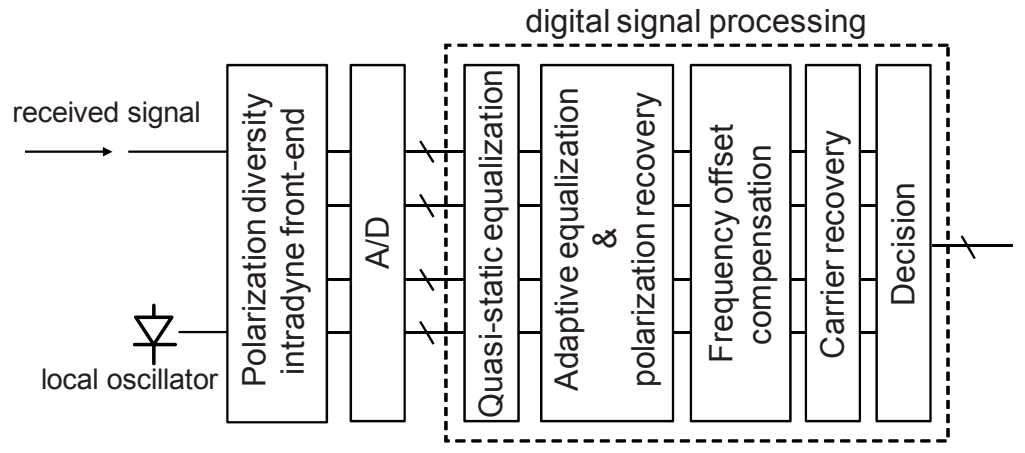

Figure 1. In general a digital coherent receiver consists of a polarization diversity optical front-end at which a received optical signal is mixed with the light of a local oscillator laser by means of optical hybrids. The resultant 4 optical signals, inphase- and quadrature components for each of the polarizations, are converted into electrical signals by photodiodes and sampled by analog-to-digital converters. The signals are then processed in the digital domain.

- Frequency offset compensation

In general the transmit- and local oscillator lasers are set to the same ITU-T grid frequency and commercially available lasers maintain that frequency to within an accuracy in the order of $\pm 2.5 \mathrm{GHz}$ or better. Therefore, the maximum frequency offset, the difference between transmit- and local oscillator laser frequencies, can be as large as $5 \mathrm{GHz}$. Carrier phase recovery algorithms like theViterbi-Viterbi $4^{\text {th }}$ power [16] require a much smaller frequency offset that is in the order of e.g only a few $10 \mathrm{MHz}$ in the case of $100 \mathrm{Gbit} / \mathrm{s}$ DP-QPSK. Frequency offset compensation achieves and maintains by means of digital signal processing such a small frequency offset.

- Carrier phase recovery

Instead of trying to synchronize the optical phase of the local laser to the carrier phase of the signal, a digital coherent receiver continuously calculates the carrier phase. The Viterbi-Viterbi algorithm is widely referred to recover the carrier phase of a QPSK signal. Data is received by means of the difference between the local laser and the signal carrier phase plus a phase of $0^{\circ}, 90^{\circ}, 180^{\circ}, 270^{\circ}$ with which the data is encoded onto the signal. Raising to the power of 4 , the data information vanishes and the carrier phase is extracted. Averaging over multiple symbols smoothes random variations due to optical amplifier's amplified spontaneous emission (ASE) noise. The extracted carrier phase is subtracted from the original received data and the resultant data concentrates around the $0^{\circ}, 90^{\circ}$, $180^{\circ}, 270^{\circ}$ data points.

- Decision

In a final stage the phase encoded data is extracted by means of threshold decisions. Differential coding may be employed as an effective countermeasure of so called cycle slips at which the received signals phase changes by more than 90 due to transmission impairments [17]. 


\section{ADVANCED ALGORITHMS}

\subsection{Improved carrier phase recovery}

The most basic functional block of a digital coherent receiver is the carrier phase recovery. Synchronization of the phase of the local oscillator laser and the phase of the received optical signal is not performed by optical means but by means of digital processing. For QPSK modulation, the phase of the carrier signal is retrieved by raising the received symbols to the power of 4 thus eliminating data information. Data information is retrieved by subtraction of the carrier phase from the received symbol. This function is shown in figure 2 as basic Viterbi-and-Viterbi carrier phase recovery.

Under the assumption that the carrier phase does not change over the duration of a certain number of symbols, averaging allows to smooth the fluctuations caused by ASE noise. If only ASE noise is considered, the more symbols are used for averaging the more exact the carrier phase is retrieved. However, phase fluctuations are also caused by other effects like frequency offset between the carrier and the local laser, and transmission impairments like cross-phase modulation (XPM). Possible means to improve the performance of the carrier phase recovery are frequency offset compensation, optimization of the number of symbols used for averaging, and weighted averaging [18] as illustrated in figure 2.

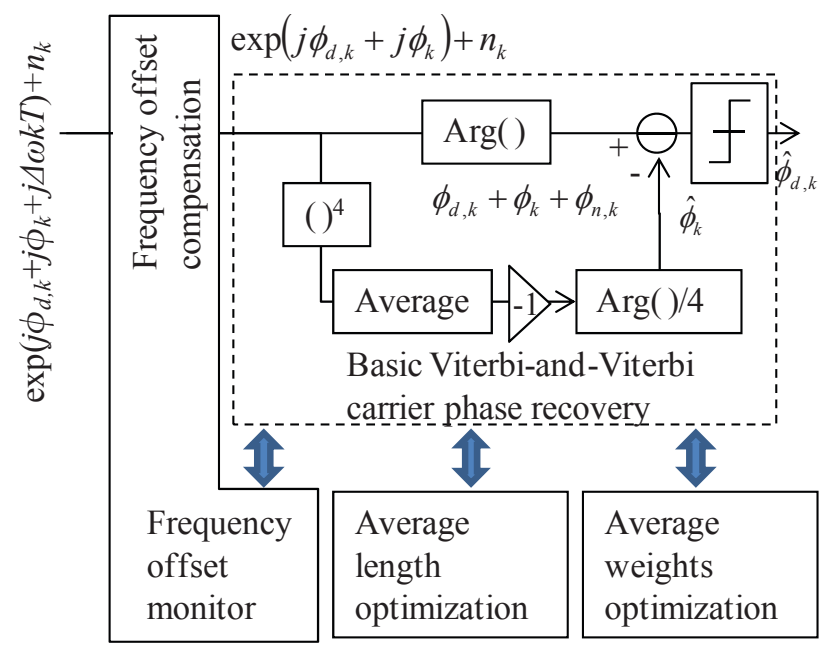

Figure 2. The basic Viterbi-and-Viterbi carrier phase recovery and potential means to improve performance like frequency offset compensation, optimization of the number of symbols used for averaging, and weighted averaging.

The basic Viterbi-and-Viterbi carrier phase recovery requires the frequency offset between the local laser and the signal carrier to be less than a few $10 \mathrm{MHz}$ in the case of $100 \mathrm{Gbit} / \mathrm{s}$ DP-QPSK [19]. Optical frequencies of commercially available lasers deviate by no more than about up to $2.5 \mathrm{GHz}$ from the ITU-T grid, the exact number depends on laser specifications. The resultant frequency offset of up to about $5 \mathrm{GHz}$ could be addressed by automatic frequency control of the local laser. It certainly is a challenge to feedback control laser frequency to within a few $10 \mathrm{MHz}$ in an economical manner. Another method that does not need a feedback loop to the local laser is based solely on digital processing. In principle frequency offset estimation can be performed by raising the received symbols to the fourth power [20]. Due to ambiguity, the detection range is limited to $\pm 1 / 8 \mathrm{~T}$ with $\mathrm{T}$ being the symbol duration. In the case of $100 \mathrm{Gbit} / \mathrm{s} \mathrm{DP}-$ QPSK that translates to an estimation range of $\pm 3.125 \mathrm{GHz}$ which might not be sufficient. It is possible to extend that range by the concept of pre-decision [21], and track frequency offset changes in a 4 times larger range of $\pm 1 / 2 \mathrm{~T}$. Figure 3 shows the principle of the pre-decision based frequency offset estimator. The parallelized architecture enabled hardware implementation and the performance was experimentally verified using a FPGA based $1 \mathrm{Gbit} / \mathrm{s}$ real-time digital coherent receiver [6]. As can be seen from the experimental results depicted in figure 3, the pre-decision based frequency estimator operates over a 0.37 times extended range when compared to an estimator based on the $4^{\text {th }}$ power calculation. 

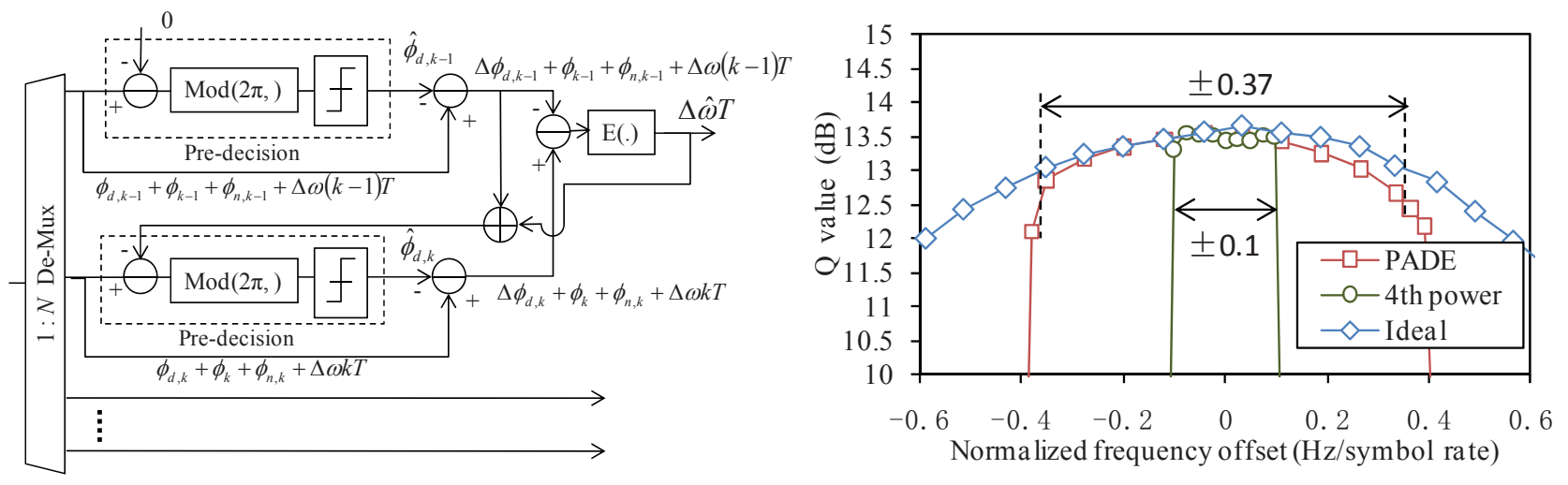

Figure 3. The parallel architecture of the pre-decision-based angle differential frequency offset estimator (left) and experimental results obtained with a $1 \mathrm{Gbit} / \mathrm{s}$ QPSK real-time digital coherent receiver demonstrating a 0.37 times extended frequency tracking range of the pre-decision-based approach (PADE) compared with the $4^{\text {th }}$-power method.

Another approach that enhances the performance of a digital coherent receiver relates to the number of symbols that are used for computing the carrier phase. Using and averaging over a larger number of symbols addresses effectively ASE noise and enhances the accuracy of the calculated carrier phase. However, effects like cross-phase modulation (XPM) change the carrier phase on a short term scale potentially in the order of only a few symbols. If too many symbols are used for averaging, the carrier phase recovery can not follow these short term effects and performance degrades. Therefore, the number of symbols needs to trade-off between addressing ASE noise (large number of symbols) and short term effects (small number of symbols). As the exact device and transmission conditions can not be known a-priori, it is desirable to adaptively change the number of symbols used for averaging. A functional block of the auto-correlation zero forcing (ACZF) that optimizes the number of symbols is shown in figure 4. The architecture allows for parallelized computation and thus can be implemented in hardware [18].

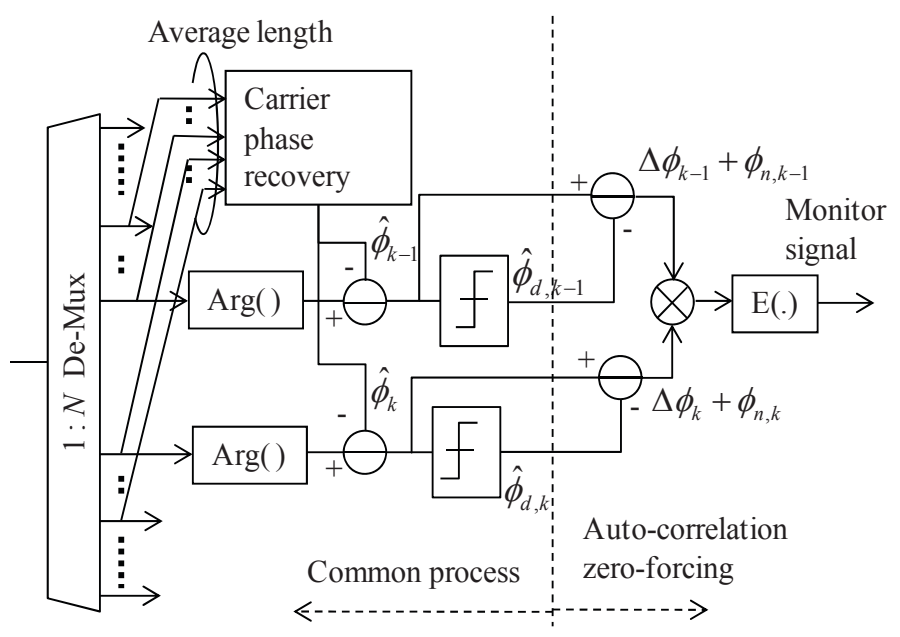

Figure 4. Parallel architecture implementation of the auto-correlation zero-forcing (ACZF) to optimize the number of symbols used for calculating the carrier phase.

Figure 5 shows results obtained with a $43 \mathrm{Gbit} / \mathrm{s}$ DP-QPSK signal received at an OSNR of $13 \mathrm{~dB}(0.1 \mathrm{~nm}$ bandwidth resolution) by means of an offline digital coherent receiver setup with processing performed using a personal computer. With an increasing number of symbols (average length) used for computation the performance of the receiver first improves, and reaches a maximum that is accompanied by a monitor signal that crosses zero. 

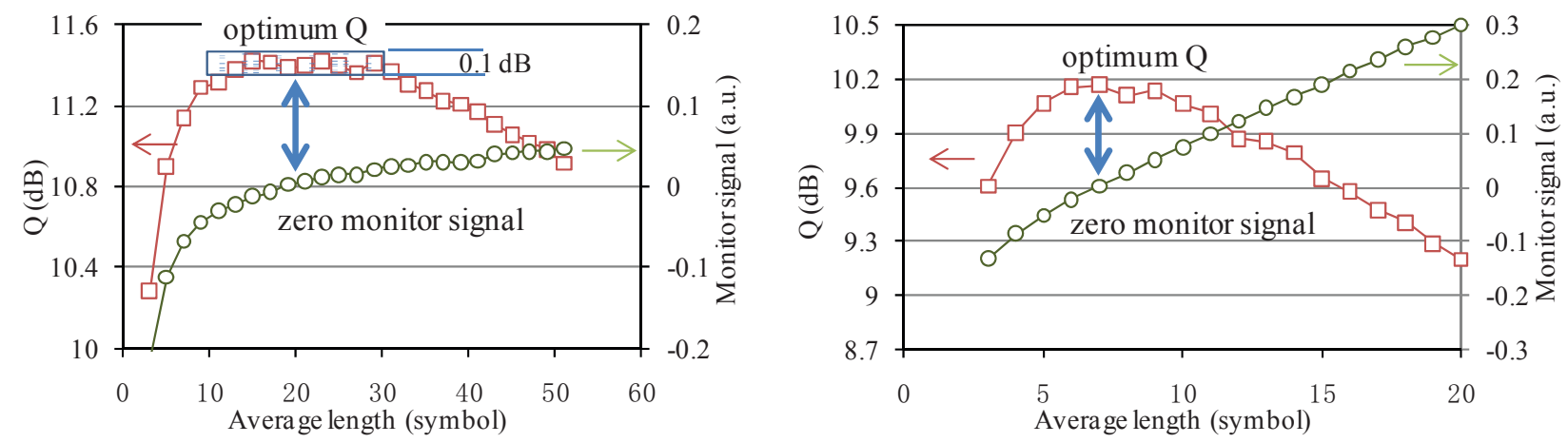

Figure 5. The performance of auto-correlation zero-forcing. Left: 43 Gbit/s DP-QPSK, back-to-back; Right: 40 ch x 100 $\mathrm{GHz}, 43 \mathrm{Gbit} / \mathrm{s}$ DP-QPSK and $10 \mathrm{Gbit} / \mathrm{s}$ OOK hybrid system after 5 x60 km transmission.

The graph on the left of figure 5 shows data obtained in a back-to-back configuration. There are no fiber transmission impairments and the receiver performance is constantly high to within a Q-value of $0.1 \mathrm{~dB}$ for an averaging length of 1030 symbols. Further increasing the number of symbols reduces the performance which can be attributed to e.g. laser phase noise and possibly frequency drift. The graph on the right of figure 5 shows data obtained after transmission through 5 spans x $60 \mathrm{~km}$ of standard single-mode fiber (SSMF), nearly 100\% dispersion compensation after each span, a fiber launch power of $0 \mathrm{dBm} / \mathrm{channel}, 100 \mathrm{GHz}$ channel spacing, a mid channel allocated to the $43 \mathrm{Gbit} / \mathrm{s}$ DP-QPSK signal, and surrounding 39 channels allocated to $10 \mathrm{Gbit} / \mathrm{s}$ on-off keying $(\mathrm{OOK})$ signals. The receiver performance as a function of the number of averaging symbols shows now a clear optimum. After having reached the optimum, the performance decreases quickly. This can be attributed to fast phase fluctuations caused by cross-phase modulation (XPM). Again, the auto-correlation zero forcing (ACZF) monitor signal crosses zero when the receiver performance reaches its maximum. That clearly indicates the possibility to automatically adapt the averaging number of symbols to maximize receiver performance in dependence on varying device- and transmission conditions.

In its simplest form, carrier phase recovery performs averaging over a certain number of symbols while each symbol is given an equal weight. Averaging weights optimization addresses the situation that the carrier phase of symbols deviates more an more from the carrier phase of the actually sampled symbol. Weights are adjusted such that high confidence symbols, those that are close to the actually sampled one, are given a high weight. This approach not only improves performance with respect to laser phase noise and laser frequency drifting but also has been shown effective to mitigate the impact of inter-channel non-linearities like cross-phase modulation.

\subsection{Inter-channel non-linearities mitigation}

Several mechanisms exist through which neighbor channels can affect a signal. For example the refractive index of an optical fiber changes with optical power causing cross-phase modulation (XPM) by which the phase and polarization of a signal is affected. Exemplary mitigation methods will be introduced in the following.

\subsubsection{Adaptively weighted filtering in the carrier phase recovery (AFCPR)}

Techniques have been proposed for carrier phase recovery that determine optimal filtering with respect to optical signalto-noise ratio (OSNR) and laser linewidth [22], and those that adaptively adjust the number of symbols used to calculate the averaged carrier phase [23]. Adaptively weighted filtering in the carrier phase recovery (AFCPR) extends these techniques, directly monitors the carrier characteristics, adapts the filter coefficients, and can mitigate the impact of cross-phase modulation (XPM). The functional block diagram of AFCPR is shown in figure 6 [24].

The effectiveness of the AFCPR is experimentally investigated in a transmission experiment. The transmission line consisted of 6 spans x $75 \mathrm{~km}$ non-zero dispersion shifted fiber (NZ-DSF) with 80\% dispersion compensation after each span. Of the 80 channels transmitted with a channel spacing of $50 \mathrm{GHz}, 75$ are $11.1 \mathrm{Gbit} / \mathrm{s}$ OOK modulated and channels at locations 5, 21, 41, 61, and 73 are $43 \mathrm{Gbit} / \mathrm{s}$ DP-QPSK with a fiber launch power for all channels of $-4 \mathrm{dBm} / \mathrm{channel}$. 


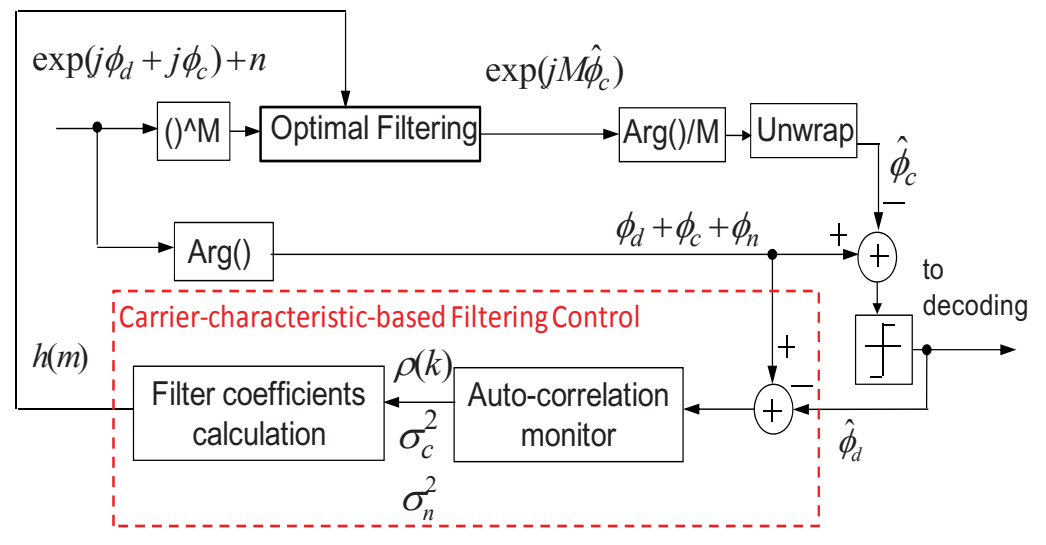

Figure 6. The principle of the adaptive filtering carrier phase recovery (AFCPR) that improves receiver performance by optimizing the weights with which symbol phases are averaged in the carrier phase recovery circuit.

The digital coherent receiver is realized with a storage oscilloscope and offline processing by means of a standard personal computer. Figure 7 shows the AFCPR monitored auto-correlation for the cases of single channel 43 Gbit/s DPQPSK transmission and the hybrid 75 channels $11.1 \mathrm{Gbit} / \mathrm{s}$ OOK and 5 channels $43 \mathrm{Gbit} / \mathrm{s}$ DP-QPSK transmission. The impact of cross-phase modulation from the neighboring $11.1 \mathrm{Gbit} / \mathrm{s}$ OOK channels is evident and the AFCPR adjusts the filter coefficient weights accordingly. On the right hand side of figure 7, the performance of AFCPR is compared with equal-weighted carrier phase recovery considering further the variants of fixed (21) and optimized number of symbols. Measurements are taken by means of the Q-value at an OSNR $(0.1 \mathrm{~nm}$ bandwidth resolution) of $13 \mathrm{~dB}$. AFCPR always performs best and becomes more advantageous with increasing number of spans.
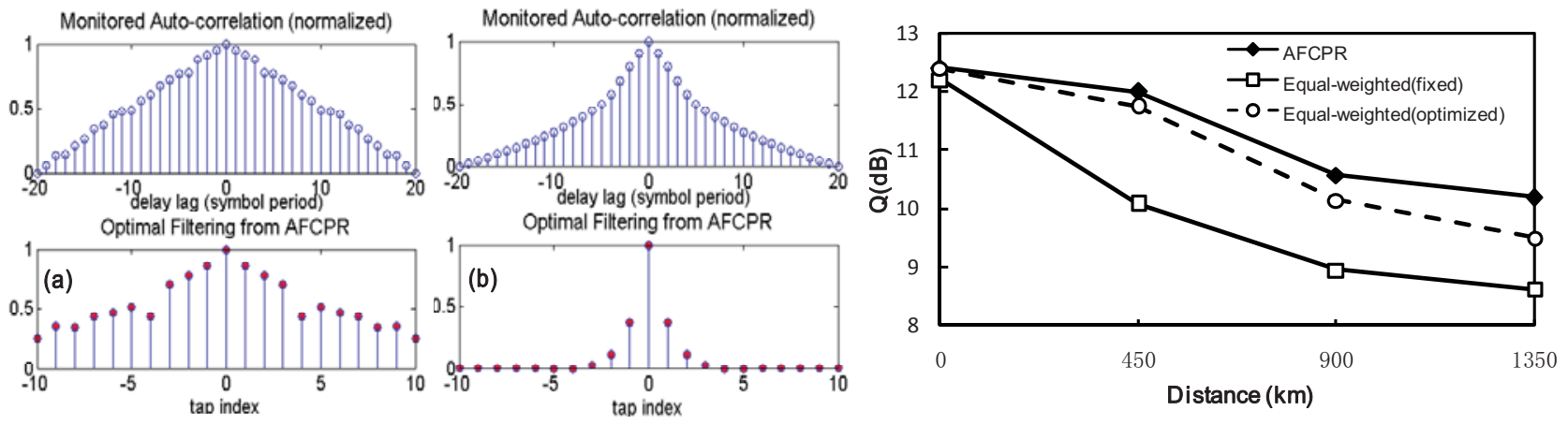

Figure 7. Performance with AFCPR and equal-weighted carrier recovery (launch power $=-4 \mathrm{dBm} / \mathrm{channel}$ and OSNR $=13$ dB). Monitored Auto-correlations and Optimal Filters((a) single channel QPSK (b) 80 channel QPSK/OOK hybrid)

\subsubsection{Non-linear polarization cross-talk canceller (NPCC)}

The cross-phase modulation impact on signal polarization does not impair a single polarization receiver (at least without optical polarization mode dispersion compensation). For dual-polarization signals, polarization scattering is induced and can have a major impact on receiver performance [25].

In an experiment as depicted in figure 8, the effect of polarization scattering is demonstrated [26]. The transmission line is comprised of 5 spans x $60 \mathrm{~km}$ standard single-mode fiber (SSMF) with 105\% dispersion compensation after each span. 40 channels of $112 \mathrm{Gbit} / \mathrm{s}$ DP-QPSK signals with a channel spacing of $100 \mathrm{GHz}$ are transported. The fiber launch power 
is $2.5 \mathrm{dBm} /$ channel. The receiver is realized with a digital storage oscilloscope and algorithms are implemented in a standard personal computer for offline processing.

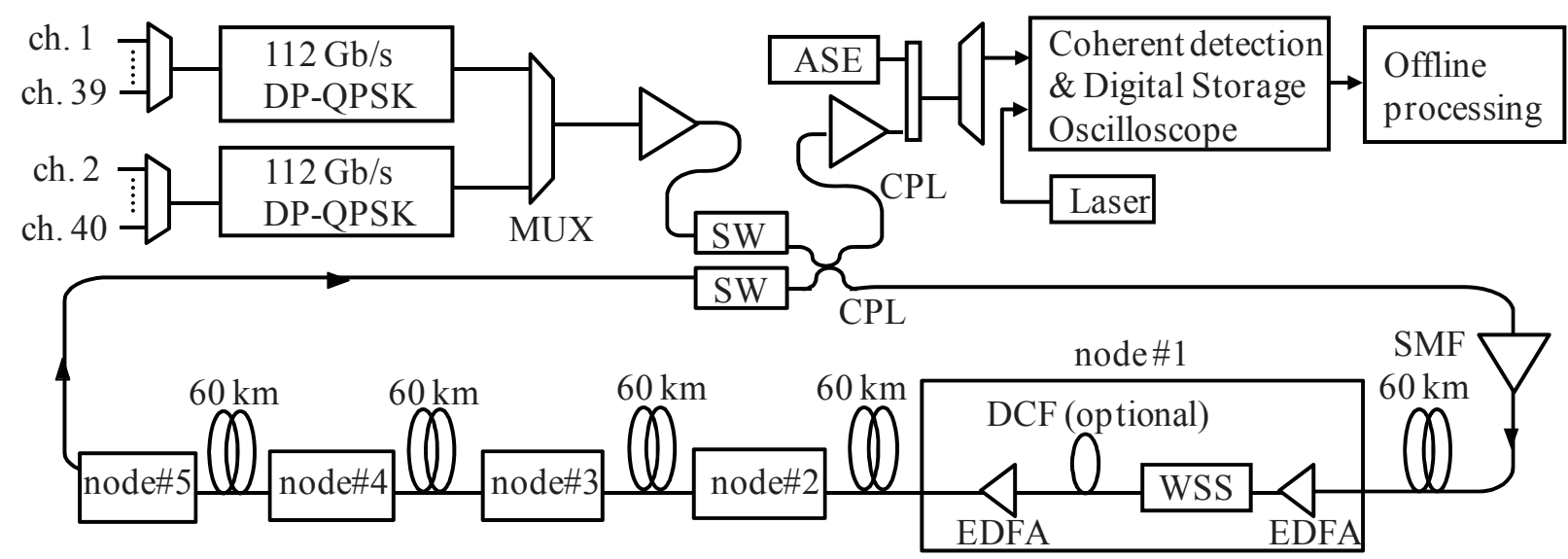

Figure 8. Transmission experiment to investigate the effect of polarization crosstalk comprised of 40 channels $112 \mathrm{Gbit} / \mathrm{s}$ DP-QPSK at $100 \mathrm{GHz}$ channel spacing, 5 spans of $60 \mathrm{~km}$ standard single-mode fiber (SSMF) with 105\% dispersion compensation, fiber launch power of $2.5 \mathrm{dBm} /$ channel and a digital coherent receiver realized with a storage oscilloscope and a standard personal computer for offline processing.

In figure 9, the measured polarization crosstalk from vertical- to horizontal polarization (Pol. V to Pol. $\mathrm{H}$ ) and vice-versa from horizontal- to vertical polarization (Pol. H to Pol. V) is shown after the signal transmitted over 0, 300, 900, and $1500 \mathrm{~km}$. It is observed that the auto-correlation length is around several tens of symbols which is indicative of polarization crosstalk changes in the order of Gigahertz. Such changes are faster than the tracking speed of typical polarization de-multiplexing circuits which operate in the order of Megahertz or less. However, polarization crosstalk changes span over several symbols and are therefore slower than the symbol rate. This allows polarization crosstalk mitigation by means of digital signal processing.
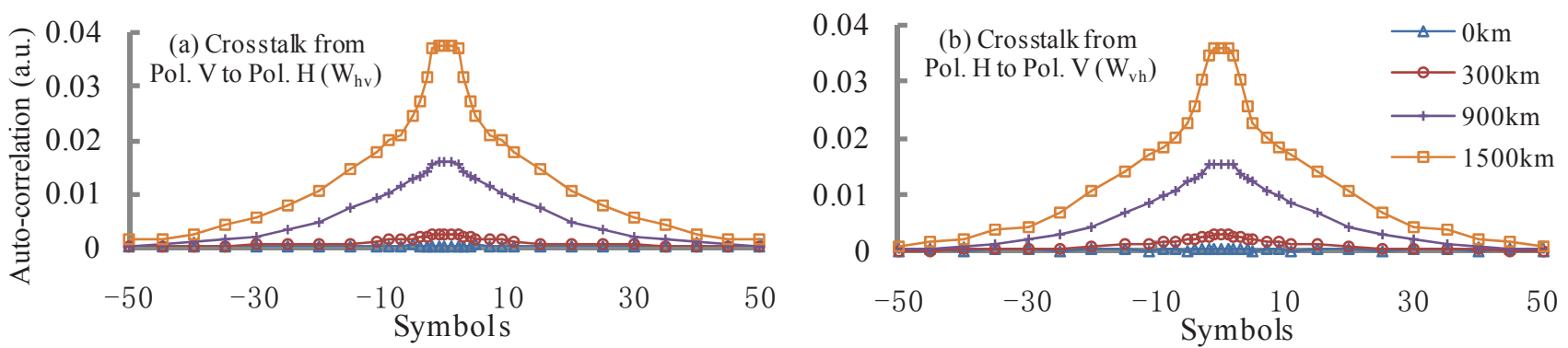

Figure 9. Measured nonlinear polarization crosstalk from vertical- to horizontal polarization (Pol. V to Pol. H) and viceversa with a transmission setup comprised of 40 channels $112 \mathrm{~Gb} / \mathrm{s}$ DP-QPSK, $60 \mathrm{~km}$ spans of standard single-mode fiber with $105 \%$ dispersion compensation, and $2.5 \mathrm{dBm} /$ channel fiber launch power. The auto-correlation length is around several tenth of symbols which indicates polarization crosstalk changes slower than the symbol-rate that in turn allows mitigation by means of digital signal processing.

The functional block diagram of a non-linear polarization crosstalk canceller (NPCC) is shown in figure 10. The polarization crosstalk factors $\mathrm{W}_{\mathrm{hv}}$ and $\mathrm{W}_{\mathrm{vh}}$ are estimated and used to remove the scattered signals from each polarization. Comparing the constellation diagrams before the NPCC and after the NPCC clearly shows the mitigation potential. 


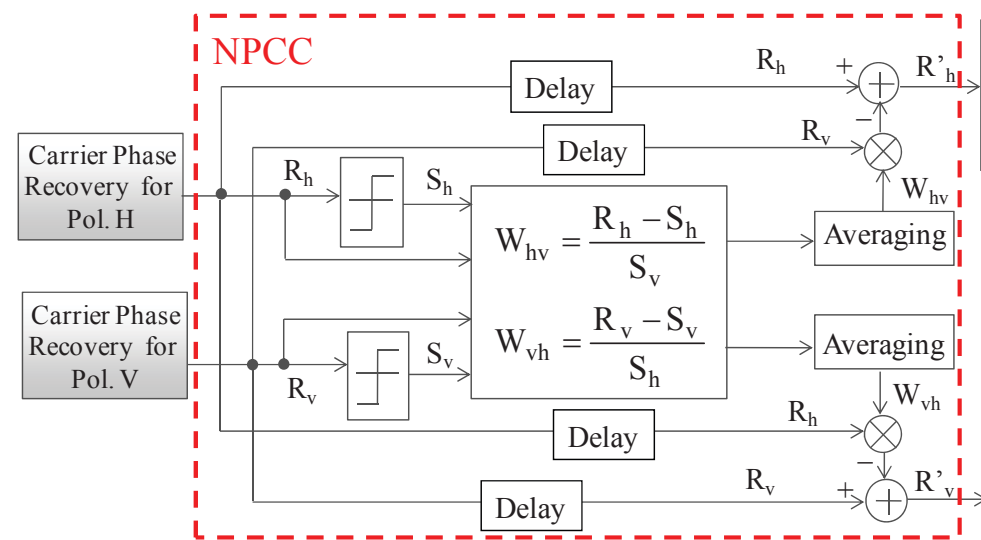

(a) Functional block diagram of the nonlinear polarization crosstalk canceller (NPCC)

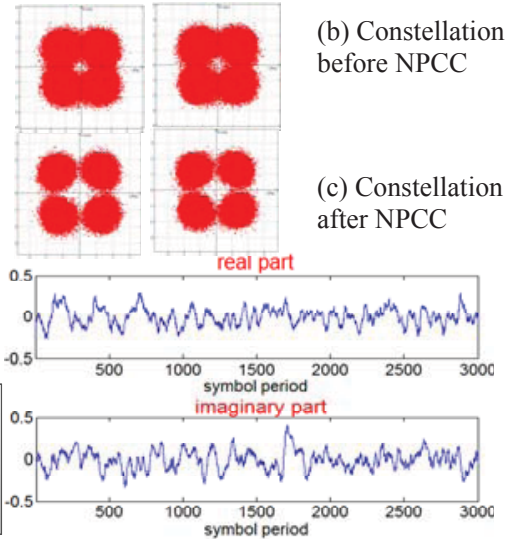

(d) Estimated polarization crosstalk factor

Figure 10. Functional block diagram of the nonlinear polarization crosstalk canceller (NPCC) that estimates the polarization crosstalk factors $\mathrm{W}_{\mathrm{hv}}$ and $\mathrm{W}_{\mathrm{vh}}$ and uses that information to remove the scattered signals from each polarization. The clearly improved constellation diagram after NPCC demonstrates the mitigation potential.

\subsection{Intra-channel non-linearities mitigation}

The most prominent intra-channel non-linear effect is self-phase modulation (SPM) by which a signal's changing optical power changes the optical fiber's refractive index and in turn impairs itself. As the bit sequence of the signal is known, equalization of intra-channel non-linearities is in principal possible. An early proposal described receiver side equalization of SPM in a travelling wave tube for satellite communications [27].

For optical communications, a multi-stage nonlinear compensator is demonstrated to be effective [28]. The functional block diagram is shown in figure 11. Multiple stages of complex FIR filters, linear equalizer (LE), and nonlinear cores, nonlinear equalizer (NLE), compensate for both the chromatic dispersion and the nonlinear phase shift accumulated at the transmission spans.

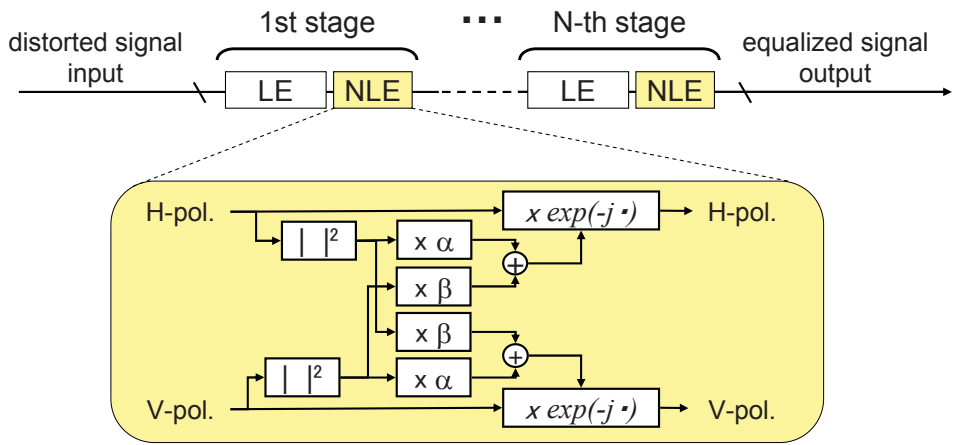

Figure 11. Schematic functional block diagram of a dual-polarization multi-stage nonlinear compensator (DP-NLC) consisting of linear equalizer (LE) that compensate for chromatic dispersion and nonlinear equalizer (NLE) that compensate for the nonlinear phase shift.

In a transmission experiment the performance of the DP-NLC is demonstrated. As depicted in figure 8, at $100 \mathrm{GHz}$ spacing, $112 \mathrm{Gbit} / \mathrm{s}$ DP-QPSK signals are transmitted through a circulating loop of 5 spans x $60 \mathrm{~km}$ standard singlemode fiber (SSMF). The digital coherent receiver is realized by means of a storage oscilloscope and offline processing with a standard personal computer.

The dependence of the Q-improvement on the number of DP-NLC segments or stages is shown in figure 12, left hand side for the case of $5.5 \mathrm{dBm} /$ channel fiber launch power, $0 \%$ dispersion compensation, after transmission through 25 spans. It is evident that the performance drastically reduces if the number of compensator stages is less than the number of transmission spans. On the right hand side of figure 12, the dependence of the Q-penalty on the dispersion compensating ratio for the cases with and without DP-NLC is shown. Always DP-NLC performs better when compared to the case without compensator. The best performance is achieved at $0 \%$ dispersion compensation ratio. 

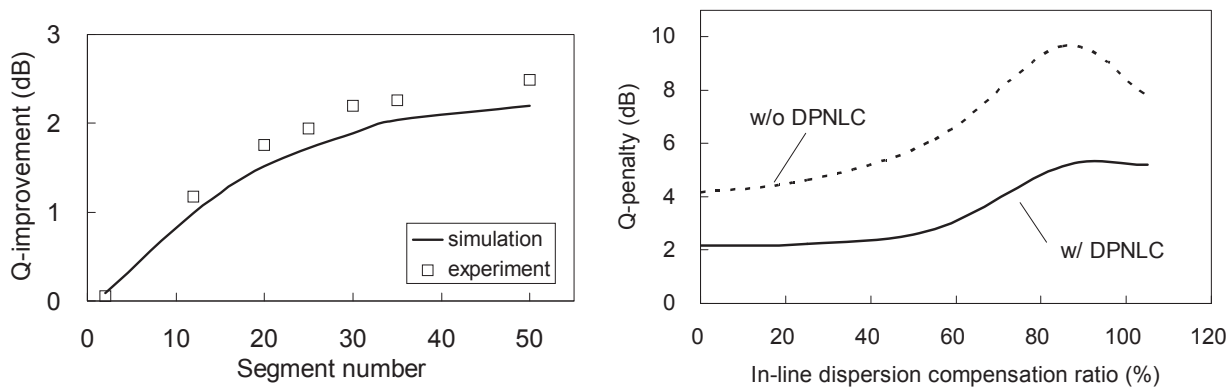

Figure 12. Left: DP-NLC compensator performance depends on the number of segments and improves until the segment number equals the transmission span number (here 25 spans). Right: Dependence of Q-penalty on the per span dispersion compensation ratio shows that DP-NLC always outperforms the case without compensator.

As shown in figure 13, the Q-penalty is significantly reduced with DP-NLC in particular for higher fiber launch powers. Effectively, with DP-NLC higher fiber launch powers are supported and in the case of the investigated transmission system, a net $\mathrm{Q}$ gain of $1.7 \mathrm{~dB}$ is achieved.
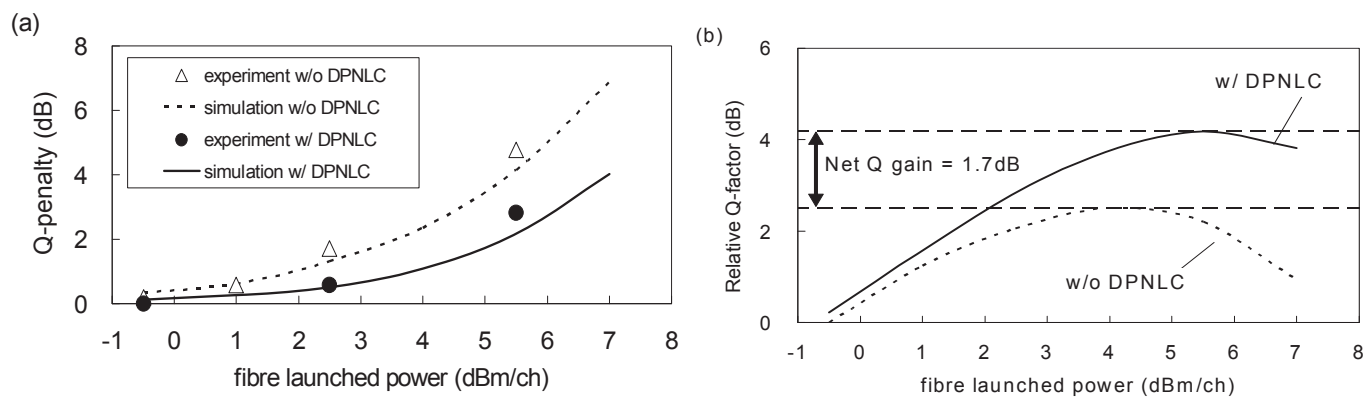

Figure 13. Compensation of non-linearities by means of the DP-NLC reduces the Q-penalty in particular for higher fiber launch powers (a). With DP-NLC a higher fiber launch is supported resulting in a net Q gain of $1.7 \mathrm{~dB}$ for the particular transmission system investigated (b).

\section{SUMMARY}

Digital coherent receiver technology enabled a jump in the bit-rate of transceivers for optical telecommunications. Based on the DP-QPSK modulation format, $100 \mathrm{Gbit} / \mathrm{s}$ transceivers have become a reality. Following the historic path of ever increasing bit-rates, one research trend focuses on technologies towards realizing $400 \mathrm{Gbit} / \mathrm{s}$ and $1 \mathrm{Tbit} / \mathrm{s}$. However, it is very desirable to improve on the performance of $100 \mathrm{Gbit} / \mathrm{s}$ digital coherent receivers. There is still a huge potential to do so by means of algorithms. Exemplary advanced algorithms that improve the carrier phase recovery by means of e.g extended range frequency offset compensation, and algorithms that address inter- and intra-channel non-linearities have been introduced and demonstrated potential to significantly improve the transceiver performance.

\section{REFERENCES}

[1] J. Winters, "Equalization in coherent lightwave systems using a fractionally spaced equalizer," J. Lightw. Technol., vol. 8, no. 9, pp. 1487-1491 (1990).

[2] S. Betti, F. Curti, G. De Marchis, and E. Iannone, "A novel multilevel coherent optical system: 4-quadrature signaling," J. Lightw. Technol., vol. 9, no. 4, pp. 514-523 (1991).

[3] F. Derr, "Coherent optical QPSK intradyne system: Concept and digital receiver receiver realization," J. Lightw. Technol., vol. 10, no. 9, pp. 1290-1296 (1992).

[4] A. Leven, N. Kaneda, A. Klein, U.-V. Koc, and Y.-K. Chen, "Real-time implementation of 4.4 Gbit/s QPSK intradyne receiver using field programmable gate array," Inst. Electr. Eng. Electron. Lett., vol. 42, pp. 1421-1422 (2006). 
[5] T. Pfau, S. Hoffmann, R. Peveling, S. Bhandare, S. K. Ibrahim, O. Adamczyk, M. Porrmann, R. Noe, and Y. Achiam, "Coherent digital polarization diversity receiver for real-time polarization-multiplexed QPSK transmission at 2.8 Gb/s," IEEE Photon. Technol. Lett., vol. 19, no. 24, pp. 1988-1990 (2007).

[6] H. Nakashima, T. Tanimura, T. Hoshida, S. Oda, J. C. Rasmussen, L. Li, Z. Tao, Y. Ishii, K. Shiota, K. Sugitani, H. Adachi, "Novel wide-range frequency offset compensator demonstrated with real-time digital coherent receiver" in Proc. ECOC 2008, paper Mo.3.D.4 (2008).

[7] H. Sun, K.-T. Wu, and K. Roberts, "Real-time measurements of a 40 Gb/s coherent system,” Opt. Exp., vol. 16, no. 2, pp. 873-879 (2008).

[8] T. Tanimura, S. Oda, T. Tanaka, T. Hoshida, Z. Tao, and J. C. Rasmussen, "A simple digital skew compensator for coherent receiver," in Proc. ECOC 2009, paper 7.3.2 (2009).

[9] C. Xie, "Suppression of inter-channel nonlinearities in WDM coherent PDM-QPSK systems using periodic-groupdelay dispersion compensators," in Proc. ECOC 2009, Paper P4.08 (2009).

[10] G. Goldfarb, M. G. Taylor, and G. Li, "Experimental demonstration of fiber impairment compensation using the split-step finite-impulse-response filtering method," IEEE Photon. Tech. Lett., vol. 20, no. 22, pp. 1887-1889 (2008).

[11] D. S. Millar, S. Makovejs, V. Mikhailov, R. I. Killey, P. Bayvel, and S. J. Savory, "Experimental comparison of nonlinear compensation in long-haul PDM-QPSK transmission at 42.7 and $85.4 \mathrm{~Gb} / \mathrm{s}$," in Proc. ECOC 2009, Paper Th9.4.4 (2009).

[12] J. C. Geyer, C. R. S. Fludger, T. Duthel, C. Schulien, and B. Schmauss, „Efficient frequncy domain chromatic dispersion compensation in a coherent Polmux QPSK-reveiver," in Proc. OFC 2010, paper OWV5 (2010).

[13] G. Ghosh, M. Endo, T. Iwasaki, "Temperature-dependent Sellmeier coefficients and chromatic dispersions for some optical fiber glass," J. Lightw. Technol., vol. 12, no. 8, pp. 1338-1342 (1992).

[14] M. El-Darawy, T. Pfau, C. Wördehoff, and R. Noe, "Performance of modified decision-directed polarization control/demultiplex algorithm in coherent receiver," in Proc. OFC/NFOEC 2010, paper JThA9 (2010).

[15] A. Leven, N. Kaneda, and Ch. Young-Kai, "A real-time CMA-based $10 \mathrm{~Gb} / \mathrm{s}$ polarization demultiplexing coherent receiver implemented in an FPGA," in Proc. OFC/NFOEC 2008, paper OTuO2 (2008).

[16] A. J. Viterbi and A. M. Viterbi, "Nonlinear estimation of PSK-modulated carrier phase with application to burst digital transmission,” IEEE Trans. on Info. Theory, vol. IT-29, No. 4, pp. 543-551 (1983).

[17] M. G. Taylor, "Phase estimation methods for optical coherent detection using digital signal processing," J. Lightw. Technol., vol. 27, no. 7, pp. 901-913 (2009).

[18] Z. Tao, L. Li, L. Liu, W. Yan, H. Nakashima, T. Tanimura, S. Oda, T. Hoshida, and J. C. Rasmussen, "Improvements to digital carrier phase recovery algorithm for high-performance optical coherent receivers," IEEE JSTQE, vol. 16, no. 5, pp. 1201-1209 (2010).

[19] L. Li, Z. Tao, S. Oda, T. Hoshida, and J. C. Rasmussen, "Wide-range, accurate and simple digital frequency offset compensator for optical coherent receivers," in Proc. OFC/NFOEC 2008, paper OWT4 (2008).

[20] A. Leven, N. Kaneda, Ut-Va Koc, and Young-Kai Chen, "Frequency estimation in intradyne reception," IEEE J. Photon. Technol. Lett., vol. 19, no. 6 pp. 366-368 (2007).

[21] Z. Tao, L. Li, A. Isomura, T. Hoshida, J. C. Rasmussen, "Multiplier-free phase recovery for optical coherent receivers," in Proc. OFC/NFOEC 2008, paper OWT2 (2008).

[22] E. Ip, and J. M. Kahn, "Feedforward carrier recovery for coherent optical communications," J. Lightw. Technol., vol. 25, no. 9, pp. 2675-2692 (2007).

[23] D. van den Borne, C. R. S. Fludger, T. Duthel, T. Wuth, E. D. Schmidt, C. Schulien, E. Gottwald, G. D. Khoe and H. de Waardt, "Carrier phase estimation for coherent equalization of 43-Gb/s POLMUXNRZ-DQPSK transmission with 10.7-Gb/s NRZ neighbors," in Proc. ECOC 2007, paper 7.2.3 (2007).

[24] L. Li, Z. Tao, L. Liu, W. Yan, S. Oda, T. Hoshida and J. C. Rasmussen "XPM tolerant adaptive carrier phase recovery for coherent receiver based on phase noise statistics monitoring”, in Proc. ECOC 2009, paper P3.16 (2009).

[25] C. Xie, "Nonlinear polarization effects and mitigation in polarization multiplexed transmission," in Proc. OFC/NFOEC 2010, paper OWE1 (2010).

[26] L. Li, Z. Tao, L. Liu, W. Yan, S. Oda, T. Hoshida, J. C. Rasmussen, "Nonlinear polarization crosstalk canceller for dual-polarization digital coherent receivers," in Proc. OFC/NFOE 2010, paper OWE3 (2010).

[27] A. Viterbi, "Nonlinear estimation of PSK-modulated carrier phase with application to burst digital transmission," IEEE Trans. on Inform. Theory, vol. 29, no. 4, pp. 543-551 (1983).

[28] T. Hoshida, T. Tanimura, S. Oda, T. Tanaka, H. Nakashima, Z. Tao, L. Li, L. Liu, W. Yan, and J. C. Rasmussen, "Recent progress on nonlinear compensation technique in digital coherent receiver," in Proc. OFC/NFOEC 2010, paper OTuE5 (2010). 